\title{
The dispersion relation of pion in nuclear matter
}

\author{
Liang-gang Liu \\ CCAST (World Laboratory), P. O. Box 8730, Beijing 100080 \\ AND \\ Department of Physics, Zhongshan University \\ Guangzhou, Guangdong 510275, P. R. China \\ Masahiro Nakano \\ University of Occupational and Environmental Health \\ Kitakyusyu 807, Japan
}

\begin{abstract}
We put forward a formalism to calculate the relativistic particle - hole and delta hole excitation polarization insertion for pion propagator by using the particle - hole antiparticle representation of nucleon and delta propagators in nuclear matter. The real and the imaginary part of the polarization insertion and the dispersion relation for pion propagator are calculated numerically. We find that the short range correlation enhances the delta - hole excitation but suppresses the particle - hole excitation, it also suppresses the pion condensation. We find that the effect of the short range correlation on the pion dispersion relation depends very much on the form of the short range correlation and the parameters involved.
\end{abstract}




\section{Introduction}

The pionic collective excitation and pion dispersion relation in nuclear matter (or in finite nuclei) is very important to many fields in nuclear physics. A typical example is the dilepton production in relativistic heavy - ion collision in the energy region of $\rho$ meson production ${ }^{1-4}$ ), where the production rate is determined by the pion dispersion relation and its derivative. The vanishing derivatives of the dispersion relation curve may lead to a huge enhancement of the dilepton production rate. In ref. $^{2}$ ), Xia et al calculated the rate by using nonrelativistic pion dispersion relation which has very good behavior for all momentum and energy. But it is not sure whether the nonrelativistic approximation is still applicable to very high energy momentum transfer region. On the other hand, as far as pion dispersion relation is concerned, the imaginary part of the polarization insertion of pion propagator should not be ignored in the calculation.

Now, it is very clear that a microscopic study of the relativistic pion dispersion relation is needed. The pion dispersion relation has been studied extensively in the nonrelativistic nuclear matter $\left.{ }^{5-7}\right)$, the nonrelativistic particle - hole $(p h)$ and delta - hole $(\Delta h)$ excitation and short range correlation via Landau - Migdal parameters $g^{\prime}$ are taken into account. The main conclusion is that the pion condensation, or the space - like branch of the pion dispersion relation in the critical region - zero energy and finite momentum, is removed by the short range correlation. Recently, there are some quasi-relativistic calculations on the $\Delta h$ excitation

of the pion propagator at finite temperature $\left.{ }^{8,9,10}\right)$. In ref. 10, Helgesson and Randrup used a so called realistic $\pi+\mathrm{NN}^{-1}+\Delta \mathrm{N}^{-1}$ model to calculate the pion dispersion relation in nuclear matter, they found a very unusual but interesting result $\left.{ }^{10}\right)$. That is, besides the usual collective modes there is a number of modes they named noncollective modes. One group of this noncollective modes shows pion condensation. These are different from the previous results. The real relativistic calculation can be found in a recent comprehensive study $\left.{ }^{11}\right)$. But as we have pointed out and will show it later that by using "Feynman" and "Density dependent" nucleon propagator and Feynman propagator for delta isobars, the formulae for the relativistic $p h$ and $\Delta h$ excitation can not be derived correctly $\left.{ }^{12,13}\right)$. We have found a new method and solved this problem ${ }^{12,13}$ ). This method has been used to study other properties of nuclear matter, such as dimesonic function ${ }^{14}$ ) and the binding energy of nuclear matter ${ }^{15,16}$ ). Our method is as follows: to express nucleon propagator in nuclear matter in terms of particle, 
hole, antiparticle propagators, and particle, antiparticle propagators for $\Delta$-isobars. Then the polarization insertion can be explicitly split as $p h$, particle - antiparticle $(N \bar{N})$ and $\Delta h$, antidelta - particle $(\bar{\Delta} N)$, delta - antiparticle $(\Delta \bar{N})$ excitations contribution. The $p h$ and $\Delta h$ excitations are finite, others are divergent and need a scheme to renormalize it. The $p h$ and $\Delta h$ are the most fundamental and important excitation modes for pion propagator in nuclear matter, the thresholds for their physical production are the lowest among all possible excitations. So it is necessary to understand their contribution to the pion propagator first. In this paper, we will study their polarization insertions, especially the imaginary part, and pion dispersion relation.

In the next sect., we will derive the formulae for the relativistic $p h$ and $\Delta h$ excitation polarization insertion. In the 3rd sect., we give the numerical calculations especially on the polarization insertion and the dispersion relation of $\pi$ meson with the emphasis on the effect of the short range correlation and their explanations. The summary and conclusions will be given in the last section.

\section{Relativistic $p h$ and $\Delta h$ excitation}

In this section, we will give the real $p h$ and $\Delta h$ excitation polarization insertion for pion propagator.

\subsection{Relativistic $p h$ excitation}

In ref. ${ }^{12}$ ), we have shown how to derive the relativistic $p h$ and $N \bar{N}$ excitation polarization insertion in nuclear matter. In the following, we will show the procedure briefly.

In nuclear matter, nucleon propagator $G(p)$ is usually written as "Feynman" propagator $G_{F}(p)$ plus "Density dependent" part $\left.G_{D}(p)^{12}\right)$. Taking pseudo-scalar (PS) $\pi N N$ coupling as an example: by using $G_{F}$ and $G_{D}$, the polarization insertion for pion propagator $\Pi^{P S}(\equiv$ $\left.\Pi_{F}^{P S}+\Pi_{D}^{P S}\right)$ can be written as follows:

$$
\begin{aligned}
\Pi_{F}^{P S}(q)= & -\frac{g_{\pi N N}^{2}}{(2 \pi)^{3}} \int \frac{d \mathbf{p}}{E_{\mathbf{p}} E_{\mathbf{p}-\mathbf{q}}}\left[4 E_{\mathbf{p}-\mathbf{q}}+\right. \\
& \left.q^{2}\left(\frac{1}{E_{\mathbf{p}}+E_{\mathbf{p}-\mathbf{q}}-q_{0}-i \epsilon}+\frac{1}{E_{\mathbf{p}}+E_{\mathbf{p}-\mathbf{q}}+q_{0}-i \epsilon}\right)\right]
\end{aligned}
$$




$$
\begin{aligned}
\Pi_{D}^{P S}(q)= & \frac{g_{\pi N N}^{2}}{(2 \pi)^{3}} \int \frac{d \mathbf{p}}{E_{\mathbf{p}} E_{\mathbf{p}-\mathbf{q}}}\left\{\left(1-n_{\mathbf{p}}\right) n_{\mathbf{p}-\mathbf{q}}\right. \\
& \cdot q^{2}\left(\frac{1}{E_{\mathbf{p}}-E_{\mathbf{p}-\mathbf{q}}-q_{0}-i \epsilon}+\frac{1}{E_{\mathbf{p}}-E_{\mathbf{p}-\mathbf{q}}+q_{0}-i \epsilon}\right) \\
& \left.+n_{\mathbf{p}}\left[4 E_{\mathbf{p}-\mathbf{q}}+q^{2}\left(\frac{1}{\cdot} E_{\mathbf{p}}+E_{\mathbf{p}-\mathbf{q}}-q_{0}-i \epsilon+\frac{1}{E_{\mathbf{p}}+E_{\mathbf{p}-\mathbf{q}}+q_{0}-i \epsilon}\right)\right]\right\},
\end{aligned}
$$

where $q=\left(q_{0}, \mathbf{q}\right)$ is four energy-momentum,円 $g_{\pi N N}$ is PS $\pi N N$ coupling constant. $E_{\mathbf{p}}=$ $\sqrt{\mathbf{p}^{2}+\tilde{m}_{N}^{2}}, \tilde{m}_{N}$ is nucleon effective mass in nuclear matter, $n_{\mathbf{p}}$ is the nucleon distribution function at zero temperature. It is obvious $\Pi_{F}^{P S}$ is divergent, it comes from the contribution of two $G_{F}$ in the integrand of the nucleon loop integration. The remaining part is finite and gives $\Pi_{D}^{P S}$. People usually omits $\Pi_{F}$ and treat $\Pi_{D}$ as $p h$ excitation. Later we will see $\Pi_{F}^{P S}$ is not due to $N \bar{N}$ excitation, neither $\Pi_{D}^{P S}$ is due to $p h$ excitation.

On the other hand, $G(p)$ can also be expressed as follows $\left.{ }^{17,18}\right)$ :

$$
\begin{gathered}
G(p)=S_{p}(p)+S_{h}(p)+S_{\bar{p}}(p) \\
S_{p}(p)=\frac{\tilde{m}_{N}}{E_{\mathbf{p}}} \frac{\left(1-n_{\mathbf{p}}\right) \Lambda_{+}(\mathbf{p})}{p_{0}-E_{\mathbf{p}}+i \epsilon} \\
S_{h}(p)=\frac{\tilde{m}_{N}}{E_{\mathbf{p}}} \frac{n_{\mathbf{p}} \Lambda_{+}(\mathbf{p})}{p_{0}-E_{\mathbf{p}}-i \epsilon}, \\
S_{\tilde{p}}(p)=-\frac{\tilde{m}_{N}}{E_{\mathbf{p}}} \frac{\Lambda_{-}(-\mathbf{p})}{p_{0}+E_{\mathbf{p}}-i \epsilon}
\end{gathered}
$$

here $\Lambda_{+}(\mathbf{p}), \Lambda_{-}(\mathbf{p})$ is the particle and antiparticle projection operator $S_{p}, S_{h}, S_{\bar{p}}$ is the particle, hole, antiparticle propagator, respectively. When these propagators are used to calculate polarization insertion, the nonvanishing parts will be $p h$ and $N \bar{N}$ excitations, that is $\Pi_{p h}^{P S}, \Pi_{N \bar{N}}^{P S}$ :

$$
\begin{aligned}
\Pi^{P S}(q)=\Pi_{p h}^{P S}(q)+\Pi_{N N}^{P S}(q), \\
\Pi_{p h}^{P S}(q)=\frac{g_{\pi N N}^{2}}{(2 \pi)^{3}} \int \frac{d \mathbf{p}}{E_{\mathbf{p}} E_{\mathbf{p}-\mathbf{q}}}\left(1-n_{\mathbf{p}}\right) n_{\mathbf{p}-\mathbf{q}}\left[2\left(E_{\mathbf{p}}-E_{\mathbf{p}-\mathbf{q}}\right)\right. \\
\left.+q^{2}\left(\frac{1}{E_{\mathbf{p}}-E_{\mathbf{p}-\mathbf{q}}-q_{0}-i \epsilon}+\frac{1}{E_{\mathbf{p}}-E_{\mathbf{p}-\mathbf{q}}+q_{0}-i \epsilon}\right)\right],
\end{aligned}
$$

\footnotetext{
${ }^{1}$ We use the conventions of Bjorken and Drell, Relativistic Quantum Fields, (McGraw-Hill, New York, 1965).
} 


$$
\begin{aligned}
\Pi_{N N}^{P S}(q)= & -\frac{g_{\pi N N}^{2}}{(2 \pi)^{3}} \int \frac{d \mathbf{p}}{E_{\mathbf{p}} E_{\mathbf{p}-\mathbf{q}}}\left(1-n_{\mathbf{p}}\right)\left[2\left(E_{\mathbf{p}}+E_{\mathbf{p}-\mathbf{q}}\right)\right. \\
& \left.+q^{2}\left(\frac{1}{E_{\mathbf{p}}+E_{\mathbf{p}-\mathbf{q}}-q_{0}-i \epsilon}+\frac{1}{E_{\mathbf{p}}+E_{\mathbf{p}-\mathbf{q}}+q_{0}-i \epsilon}\right)\right],
\end{aligned}
$$

The sum of $\Pi_{p h}^{P S}$ and $\Pi_{N N}^{P S}$ is equal to sum of $\Pi_{F}^{P S}$ and $\Pi_{D}^{P S}$. Since $\Pi_{p h}^{P S}$ is not equal to $\Pi_{D}^{P S}$, $\Pi_{N N}^{P S}$ is not equal to $\Pi_{F}^{P S}$, so $\Pi_{F}^{P S}$ does not stand for $N \bar{N}$ excitation, neither $\Pi_{D}^{P S}$ stands for $p h$ excitation. That means one can not get the correct expressions for $p h$ and $N \bar{N}$ excitations if $G_{F}, G_{D}$ propagators are used to calculate the polarization insertion.

Similarly, in case of pseudo-vector (PV) $\pi N N$ coupling, the $p h$ and $N \bar{N}$ excitation polarization insertions $\Pi_{p h}^{P V}, \Pi_{N N}^{P V}$ can be written as follows:

$$
\begin{aligned}
\Pi_{p h}^{P V}(q)= & \frac{2 f_{\pi N N}^{2}}{(2 \pi)^{3} m_{\pi}^{2}} \int \frac{d \mathbf{p}}{E_{\mathbf{p}} E_{\mathbf{p}-\mathbf{q}}}\left(1-n_{\mathbf{p}}\right) n_{\mathbf{p}-\mathbf{q}}\left\{\left(E_{\mathbf{p}-\mathbf{q}}-E_{\mathbf{p}}\right)\left[\left(E_{\mathbf{p}}+E_{\mathbf{p}-\mathbf{q}}\right)^{2}-\mathbf{q}^{2}\right]\right. \\
+ & \left.2 \tilde{m}_{N}^{2} q^{2}\left(\frac{1}{E_{\mathbf{p}}-E_{\mathbf{p}-\mathbf{q}}-q_{0}-i \epsilon}+\frac{1}{E_{\mathbf{p}}-E_{\mathbf{p}-\mathbf{q}}+q_{0}-i \epsilon}\right)\right\}, \\
\Pi_{N N}^{P V}(q)= & \frac{2 f_{\pi N N}^{2}}{(2 \pi)^{3} m_{\pi}^{2}} \int \frac{d \mathbf{p}}{E_{\mathbf{p}} E_{\mathbf{p}-\mathbf{q}}}\left(1-n_{\mathbf{p}}\right)\left\{\left(E_{\mathbf{p}}+E_{\mathbf{p}-\mathbf{q}}\right)\left[\left(E_{\mathbf{p}}-E_{\mathbf{p}-\mathbf{q}}\right)^{2}-\mathbf{q}^{2}\right]\right. \\
& \left.-2 \tilde{m}_{N}^{2} q^{2}\left(\frac{1}{E_{\mathbf{p}}+E_{\mathbf{p}-\mathbf{q}}-q_{0}-i \epsilon}+\frac{1}{E_{\mathbf{p}}+E_{\mathbf{p}-\mathbf{q}}+q_{0}-i \epsilon}\right)\right\},
\end{aligned}
$$

here $f_{\pi N N}=0.988$ is PV $\pi N N$ coupling constant $\left.{ }^{11}\right)$. The analytic expression for the real and the imaginary part of eq. (10) can be found in ref. ${ }^{12}$ ).

\subsection{Relativistic $\Delta h$ excitation}

The delta baryon Feynman propagator $S^{\mu \nu}(p)$ can be expressed in terms of particle and antiparticle propagators, that is $S_{\Delta}^{\mu \nu}(p)$ and $S_{\bar{\Delta}}^{\mu \nu}(p)$ :

$$
\begin{gathered}
S^{\mu \nu}(p)=S_{\Delta}^{\mu \nu}(p)+S_{\bar{\Delta}}^{\mu \nu}(p), \\
S_{\Delta}^{\mu \nu}(p)=\frac{\tilde{m}_{\Delta}}{E_{\Delta}(\mathbf{p})} \cdot \frac{\Lambda_{+}^{\mu \nu}(\mathbf{p})}{p_{0}-E_{\Delta}(\mathbf{p})+i \epsilon}, \\
S_{\bar{\Delta}}^{\mu \nu}(p)=-\frac{\tilde{m}_{\Delta}}{E_{\Delta}(\mathbf{p})} \cdot \frac{\Lambda_{-}^{\mu \nu}(-\mathbf{p})}{p_{0}+E_{\Delta}(\mathbf{p})-i \epsilon},
\end{gathered}
$$

here $E_{\Delta}(\mathbf{p})=\sqrt{\mathbf{p}^{2}+\tilde{m}_{\Delta}^{2}}, \tilde{m}_{\Delta}$ is effective mass of $\Delta$-isobars in nuclear matter. (Here we implicitly assume there is no real $\Delta$-isobars in the ground state, otherwise the decay of a real 
$\Delta$-isobar will cause the instability of nuclear matter $\left.{ }^{19}\right)$, for this reason we can omit the width of $\Delta$-isobars.) $\Lambda_{+}^{\mu \nu}(\mathbf{p}), \Lambda_{-}^{\mu \nu}(\mathbf{p})$ is particle and antiparticle projection operator, respectively,

$$
\begin{gathered}
\Lambda_{+}^{\mu \nu}(\mathbf{p})=-\frac{\gamma \cdot p+\tilde{m}_{\Delta}}{2 \tilde{m}_{\Delta}} \cdot\left[g^{\mu \nu}-\frac{1}{3} \gamma^{\mu} \gamma^{\nu}-\frac{2}{3 \tilde{m}_{\Delta}^{2}} p^{\mu} p^{\nu}+\frac{1}{3 \tilde{m}_{\Delta}}\left(p^{\mu} \gamma^{\nu}-p^{\nu} \gamma^{\mu}\right)\right], \\
\Lambda_{-}^{\mu \nu}(\mathbf{p})=-\frac{-\gamma \cdot p+\tilde{m}_{\Delta}}{2 \tilde{m}_{\Delta}} \cdot\left[g^{\mu \nu}-\frac{1}{3} \gamma^{\mu} \gamma^{\nu}-\frac{2}{3 \tilde{m}_{\Delta}^{2}} p^{\mu} p^{\nu}-\frac{1}{3 \tilde{m}_{\Delta}}\left(p^{\mu} \gamma^{\nu}-p^{\nu} \gamma^{\mu}\right)\right],
\end{gathered}
$$

here $p=\left(E_{\Delta}(\mathbf{p}), \mathbf{p}\right)$. There is a discrepancy on the overall sign for the free Lagrangian or the propagator of $\Delta$-isobar ${ }^{20}$ ). We found and checked that an overall negative sign is very important to keep the imaginary part of $\Delta h$ excitation polarization insertion negative.

The $\pi N \Delta$ interaction is given as follows $\left.{ }^{11}\right)$ :

$$
\mathcal{L}_{\pi N \Delta}=\frac{f_{\pi N \Delta}}{\sqrt{2} m_{\pi}} \bar{\psi}_{\Delta}^{\mu}\left(g_{\mu \nu}+\xi \gamma_{\mu} \gamma_{\nu}\right) \vec{T} \cdot \psi_{N} \partial^{\nu} \vec{\pi}+\text { h.c. }
$$

here $f_{\pi N \Delta}=2 f_{\pi N N}, \xi$ is an arbitrary constant ${ }^{20,21}$ ). It will contribute to the processes in which $\Delta$-isobar is off-shell. Since $\gamma_{\mu} \Lambda_{+}^{\mu \nu}(\mathbf{p})=\Lambda_{+}^{\mu \nu}(\mathbf{p}) \gamma_{\nu}=0$, so $\xi$ will not appear in the polarization function.

The nucleon-delta excitation is a very important channel to $\pi$ propagator, it is nothing but $\Delta h, \Delta \bar{N}, \bar{\Delta} N$ excitation. In this paper we only consider $\Delta h$ excitation. The excitation of other two channels require higher energy. Using $S_{h}, S_{\Delta}^{\mu \nu}, \mathcal{L}_{\pi N \Delta}$ and Feynman rules, $\Delta h$ polarization insertion can be given as follows:

$$
\begin{aligned}
\Pi_{\Delta h}\left(q_{0}, \mathbf{q}\right)= & -\frac{4 f_{\pi N \Delta}^{2}}{9 \tilde{m}_{\Delta}^{2} m_{\pi}^{2}} \int \frac{d \mathbf{p}}{E_{\Delta}(\mathbf{p}) E_{\mathbf{p}-\mathbf{q}}} n_{\mathbf{p}-\mathbf{q}}\left\{2 ( E _ { \Delta } ( \mathbf { p } ) - E _ { \mathbf { p } - \mathbf { q } } ) \left[E_{\Delta}(\mathbf{p}) E_{\mathbf{p}-\mathbf{q}} q_{0}^{2}\right.\right. \\
& \left.+\tilde{m}_{\Delta}^{2} q^{2}-(\mathbf{p} \cdot \mathbf{q})^{2}\right]-2\left(\tilde{m}_{\Delta}^{2}-\tilde{m}_{N}^{2}-\mathbf{q}^{2}\right) E_{\Delta}(\mathbf{p}) q_{0}^{2}+\left[\left(\tilde{m}_{\Delta}+\tilde{m}_{N}\right)^{2}-q^{2}\right] \\
& {\left[\frac{1}{2}\left(\left(E_{\Delta}(\mathbf{p})+E_{\mathbf{p}-\mathbf{q}}\right)^{2}+q_{0}^{2}\right)\left(E_{\Delta}(\mathbf{p})-E_{\mathbf{p}-\mathbf{q}}\right)+\left(\tilde{m}_{N}^{2}+\mathbf{q}^{2}-\tilde{m}_{\Delta}^{2}\right)\left(E_{\Delta}(\mathbf{p})+E_{\mathbf{p}-\mathbf{q}}\right)\right] } \\
& +\left[q^{2}-\left(\tilde{m}_{\Delta}+\tilde{m}_{N}\right)^{2}\right]\left[\tilde{m}_{\Delta}^{2} q^{2}-\frac{1}{4}\left(\tilde{m}_{\Delta}^{2}-\tilde{m}_{N}^{2}+q^{2}\right)^{2}\right] \\
& \left.\left(\frac{1}{E_{\Delta}(\mathbf{p})-E_{\mathbf{p}-\mathbf{q}}-q_{0}-i \epsilon}+\frac{1}{E_{\Delta}(\mathbf{p})-E_{\mathbf{p}-\mathbf{q}}+q_{0}-i \epsilon}\right)\right\} .
\end{aligned}
$$

As we have mentioned above, $\xi$ does not appear. But this is not the case if we use $G_{D}$ and Feynman propagator $\left.S^{\mu \nu 11}\right)$.

The short range correlation between $N N, N \Delta, \Delta \Delta$ can be included via Landau-Migdal parameters $g_{N N}^{\prime}, g_{N \Delta}^{\prime}, g_{\Delta \Delta}^{\prime}$. But the way of constructing short range correlation interacting vertex and intermediate state propagator is not unique ${ }^{12}$ ). Here we will use the method as other 
papers used $\left.{ }^{2,11}\right)$. Taking into account this effect, the polarization insertion $\Pi^{\prime}$ which includes $p h, \Delta h$ excitation and short range correlation in the Random Phase Approximation, can be written as follows $\left.{ }^{11}\right)$ :

$$
\Pi^{\prime}(q)=q^{2} \frac{q^{2}\left(\Pi_{p h}^{P V}(q)+\Pi_{\Delta h}(q)\right)+\Pi_{p h}^{P V}(q)\left(g_{N N}^{\prime}+g_{\Delta \Delta}^{\prime}-2 g_{N \Delta}^{\prime}\right) \Pi_{\Delta h}(q)}{\left(q^{2}+g_{N N}^{\prime} \Pi_{p h}^{P V}(q)\right)\left(q^{2}+g_{\Delta \Delta}^{\prime} \Pi_{\Delta h}(q)\right)-g_{N \Delta}^{\prime 2} \Pi_{p h}^{P V}(q) \cdot \Pi_{\Delta h}(q)} .
$$

The magnitude of $g_{N N}^{\prime}, g_{N \Delta}^{\prime}, g_{\Delta \Delta}^{\prime}$, however, is not well determined. Usually $g_{N N}^{\prime}$ is larger than $g_{N \Delta}^{\prime}$ and $\left.g_{\Delta \Delta}^{\prime}{ }^{2,10,11}\right)$. In nonrelativistic approach, $g_{N N}^{\prime}=0.9, g_{N \Delta}^{\prime}=g_{\Delta \Delta}^{\prime}=0.6$ in ref. $\left.{ }^{2}\right), g_{N N}^{\prime}$ $=0.9, g_{N \Delta}^{\prime}=0.38, g_{\Delta \Delta}^{\prime}=0.35$ in ref. $\left.{ }^{10}\right)$. But in the relativistic approach, $g_{N N}^{\prime}=g_{N \Delta}^{\prime}=g_{\Delta \Delta}^{\prime}$ $=0.6$ is used in the calculation ref. ${ }^{11}$ ), we adopt this prescription in this paper.

\section{Results and discussion}

In this section, we will give the numerical results for the real and the imaginary part of the polarization insertion and pion dispersion relation. The nucleon effective mass in the formula is determined by the Walecka's model in the relativistic Hartree approximation $(R H A)$, namely, $\frac{\tilde{m}_{N}}{m_{N}}=0.72$ for nuclear matter density $\rho=\rho_{0}\left(k_{F}=1.42 \mathrm{fm}^{-1}\right)$. A remark on the effective mass of $\Delta$-isobars $\tilde{m}_{\Delta}$ is as follows: The origin of the effective mass of $\Delta$-isobars comes from the interaction between $\sigma$ meson and $\Delta$-isobars ${ }^{11,16}$ ) (This statement is model dependent, there are other sources probably.). Due to this interaction, the $\Delta$-isobars will contribute to the binding energy as well as the effective mass of nucleon at saturation density in the 1 - loop level or higher orders. Since the nucleon effective mass is determined in the $R H A$ without $\Delta$-isobars, so for consistency we choose $\tilde{m}_{\Delta}=m_{\Delta}$ in the numerical calculation. There will be no any other free parameters.

Shown in Fig. 1 is the real part of the polarization insertion function. They

\section{Figure 1}

are all negative in this case. In the limit $\mathbf{q} \rightarrow 0, \Pi_{p h}^{P V} \rightarrow 0$ is as expected, but $\Pi_{\Delta h}$ does not vanish. This nonvanishing value will contribute to the pion dispersion relation at the point $\mathbf{q} \rightarrow 0$. Another evident feature we can observe is that the $p h$ excitation contribution is suppressed when short range correlation effect sets in. This suppression can also been seen in the imaginary part of the polarization insertion.

The imaginary part of $-\Pi^{\prime}$ at density $\rho_{0}\left(k_{F}=1.42 \mathrm{fm}^{-1}\right)$ is shown in Fig. 2 and Fig. 3. In Fig. 2, $-\Pi^{\prime}$ is shown as a function of 
\begin{tabular}{|l|l|}
\hline Figure 2 & Figure 3 \\
\hline
\end{tabular}

energy $\omega$ for fixed momentum $q=2.5 k_{F}$ (approximately $5 m_{\pi}$ ). The first peak which starts from $\omega \sim 0.4 m_{\pi}$ ends at $\omega \sim 3.2 m_{\pi}$ corresponds to the $p h$ excitation, the $\Delta h$ excitation is represented by the second peak starts at $\omega \sim 4 m_{\pi}$ ends at $\omega \sim 6 m_{\pi}$, these values correspond to the boundary of the hatch region at $q \sim 5 m_{\pi}$ in Fig. 4. It is evident that the short range correlation effect suppresses the $p h$ excitation but enhances the $\Delta h$ excitation. This is the same conclusion we have drawn from Fig. 1. The same is true for Fig. 3 where $\omega=5 m_{\pi}$ is fixed, and the first peak (dotted and solid line curves) stands for $\Delta h$ excitation while the second peak (dotted and solid line curves) stands for ph excitation. There is no zero value between peaks in this case, because the $p h$ and $\Delta h$ excitation overlap for $\omega=5 m_{\pi}, q$ in $7 m_{\pi} \sim 8 m_{\pi}$ as shown in Fig. 4. Again, the $\Delta h$ excitation is enhanced and the $p h$ excitation is suppressed by the short range correlation.

The pion dispersion relation in nuclear matter is determined by the trajectory of the poles of pion propagator, that is:

$$
\omega^{2}-q^{2}-m_{\pi}^{2}-\operatorname{Re}^{\prime}(\omega, q)=0
$$

here Re means taking the real part of $\Pi^{\prime}(\omega, q)$, it includes the contributions both from the real and the imaginary part of $\Pi_{p h}^{P V}$ and $\Pi_{\Delta h}$. Firstly, we calculate the dispersion relation in case without short range correlation effect, that is $g^{\prime}=0$, the result is shown in Fig. 4. We can see that there are three disconnected curves indicated by

Figure 4

letters $\mathbf{a}, \mathbf{b}, \mathbf{c}$. The existence of curve a implies the pion condensation happens at relatively larger momentum at about $5.6 m_{\pi}$. The upper part of curve $\mathbf{b}$ is pion-like pion mode, the lower part starts from about $q \sim 0.3 m_{\pi}$ which is mainly due to $p h$ excitation, is noncollective $p h^{10}$ ). These two modes connect at higher momentum and energy. The noncollective ph excitation mode falls into the hatched region completely thus it is damped. The curve $\mathbf{c}$ is noncollective $\Delta h$ excitation mode, because it is mainly due to $\Delta h$ excitation contribution. It disappears at $q \sim 2.6 m_{\pi}$.

When short range correlation sets in, the dispersion relation changes very much as shown in Fig. 5. There is no counterpart of curve e and the lower part of curve $\mathbf{f}$ 
in Fig. 4, and new modes appear also. The curve $\mathbf{f}$ is the compressed one of curve $\mathbf{c}$ in Fig. 4 by short range correlation. The short range correlation also brings about the new modes curve $\mathbf{d}$ and the upper part of curve $\mathbf{g}$ which are damped by $\Delta h$ decays.

We have seen the short range correlation effect is very larger indeed, however, it is not well defined on the other hand ${ }^{12}$ ). An alternative way to construct the short range correlation to the polarization insertion is as follows ${ }^{8,12}$ :

$$
\Pi^{\prime}(q)=\mathbf{q}^{4} \frac{\Pi_{p h}^{P V}(q)+\Pi_{\Delta h}(q)}{\left(-\mathbf{q}^{2}+g^{\prime} \Pi_{p h}^{P V}(q)\right)\left(-\mathbf{q}^{2}+g^{\prime} \Pi_{\Delta h}(q)\right)-g^{\prime 2} \Pi_{p h}^{P V}(q) \cdot \Pi_{\Delta h}(q)},
$$

that is to replace the $q^{2}$ in eq. (19) by $-q^{2}$. Substitute eq. (21) into eq. (20), we get the dispersion relation shown in Fig. 6. In this case, the

Figure 6

pion mode starts at $\omega=1 m_{\pi}$, because the total polarization vanishes when $\mathbf{q} \rightarrow 0$. The free pion dispersion relation (dashed line curve) above the full pion mode implies that the pion effective mass in nuclear matter is reduced comparing to the free one as a function of energy and momentum. The small island in the figure is mainly due to noncollective $\Delta h$ excitation and short range correlation. Compare Fig. 5 with Fig. 6, we can see that the short range correlation has a very large effect on the pion dispersion relation. Our studies also show that the pion dispersion relation is very sensitive to other parameters in the model, such as the effective mass of nucleon and $\Delta$-isobars, Landau-Migdal parameters and nuclear density.

The spectral function of pion $A_{\pi}$ in a $\Delta h$ model at zero and finite temperature in the nonrelativistic approach was calculated approximately ${ }^{8}$ ) and self-consistantly ${ }^{9}$ ). It is defined as follows:

$$
A_{\pi}(\omega, q)=-\frac{1}{\pi} \operatorname{I} m \frac{1}{\omega^{2}-q^{2}-m_{\pi}^{2}-\Pi^{\prime}(\omega, q)}
$$

here the polarization insertion including short range correlation effect is given by eq. (19) and eq. (20). $A_{\pi}(\omega, q)$ plotted as functions of $\omega$ for fixed $q=2.5 k_{F}$ is shown in Fig. 7 . Similar to Fig. 2, for polarization eq. (19), the short

Figure 7 
range correlation suppresses the $p h$ but enhance the $\Delta h$ excitation. The zero points in the denominator of eq. (22) gives the peaks in spectral function. But for the polarization eq. (20), the peaks disappear for those energy and momentum. For ph excitation, its effect is similar to eq. (19), but for $\Delta h$ excitation the short range correlation effect is very small. So it's very clear that the effect of short range correlation depends on its forms, and this effect can change the pion dispersion relation and other observable very much.

\section{Summary and conclusions}

In summary of this study, we have derived the real $p h$ and $\Delta h$ excitation polarization insertion for pion propagator by employing particle, hole, antiparticle propagators representation of nucleon and $\Delta$-isobars, instead of their "Density dependent" and "Feynman" propagators. The ph and $N \bar{N}, \Delta h$ and $\Delta \bar{N}, \bar{\Delta} N$ excitations are separated. The real and the imaginary part of the total polarization insertion including $p h, \Delta h$ excitations and short range correlation is calculated. We found that the short range correlation largely suppresses the ph excitation but enhances the $\Delta h$ excitation very much. The pion dispersion relations are quite different from those calculated in the nonrelativistic approximation ${ }^{2,8}$. The short range correlation has a large effect on all branches of the pion dispersion relation. The pion condensation at momentum $q \sim 5.6 m_{\pi}$ disappears when short range correlation sets in.

We have also studied the effect of the different forms of short range correlation on the pion dispersion relation and pion spectral function. The difference between two forms of short range correlation is very large. We have found but have not shown that the pion dispersion relation depends very much on the effective mass of nucleon and $\Delta$-isobar and Landau-Migdal parameters. These problem suggests that the short range correlation itself needs a careful study, and only until it is well defined, we can have the definite answer to the pion dispersion relation.

The author Liu is very grateful to E. Oset, Huan-Qing Chiang and Cheng-Guang Bao for very valuable discussions. 


\section{Reference}

1) C. Gale And J. Kopusta, Phys. Rev. C35(1987) 2107

2) L. H. Xia, C. M. Ko, L. Xiong and J. Q. Wu, Nucl. Phys. A485(1988) 721

3)G. Chanfray And P. Schuck, Nucl. Phys. A555(1993) 329

4) C. Y. Wong, Introduction to High - Energy Heavy - ion Collisions, (World Scientific, Singapore, 1994)

5) E. Oset, H. Toki and W. Weise, Phys. Rept. 83(1982) 281

6) C. Garcia - Pecio, E. Oset, L. L. Salcedo, Phys. Rev. C37(1988) 194

7) A. B. Migdal, E. E. Saperstein, M. A. Troitsky and D. N. Voskresensky, Phys. Rep. 192(1990) 179

8) P. A. Henning, H. Umezawa, Nucl. Phys. A571(1994) 617

9) C. L. Korpa, R. Malfliet, Phys. Rev. C52(1995) 2756

10)J. Helgesson And J. Randrup, Phys. Rev. C52(1995)427

11) T. Herbert, K. Wehrberger, And F. Beck, Nucl. Phys. A541 (1993) 699

12) L. G. Liu, Phys. Rev. C51(1995)3421

13) L. G. Liu, Q. X. Ma, And H. Q. Chiang, High Energy Phys. \& Nucl. Phys. 18(1994)391

14) L. G. Liu, Q. F. Zhou and T. S. Lai, Phys. Rev. C51 (1995)R2302

15) L. G. Liu, The binding energy of relativistic particle-hole in nuclear matter in the relativistic $\sigma-\omega$ model, Jou. Phys. (London) G22 (1996) 1799

16) L. G. Liu, Q. F. Zhou, The study of the binding energy of nuclear matter in the relativistic $\sigma-\omega-\pi$ model with $\Delta$-isobar degree of freedom, to be appear in Z. Phys. A

17) B. D. Serot And J. D. Walecka, Adv. in Nucl. Phys. 16(1986) 1

18) W. Bentz, A. Arima, H. Hyuga, K. Shimizu, And K. Yazaki, Nucl. Phys. A 436(1985) 593 
19) K. Wehrberger, Phys. Rept. 225(1993)272

20) see, e. g., H. B. Tang And P. J. Ellis, Redundancy of $\Delta$-isobar parameters in effective field theories, preprint NUC-MINN-96/4-T, and references therein.

21) L. M. Nath, B. Etemadi and J. P. Kimel, Phys. Rev. D3(1971) 2153 


\section{Figure Captions}

Figure 1. The real part of the polarization insertion $-\operatorname{Re} \Pi(\omega=0, q)$ (in unit of $\left.m_{\pi}^{2}\right)$ as a function of momentum $q$ (in unit of $m_{\pi}$ ) for density $\rho_{0}$. The $p h$ and $\Delta h$ contributions are dotted and dashed line. "ph $+\Delta \mathrm{h}+\mathrm{SRC} "$ is the total result by eq. (19).

Figure 2. The imaginary part of - $\Pi^{\prime}(\omega, q)$ (in unit of $m_{\pi}^{2}$ ) as a function of $\omega$ (in unit of $m_{\pi}$ ) for $\mathrm{q}=2.5 k_{F} \cdot g^{\prime}=0$, dotted line; $g^{\prime}=0.6$, solid line.

Figure. 3. - $\Pi^{\prime}\left(\omega=5 m_{\pi}, q\right)$ (in unit of $\left.m_{\pi}^{2}\right)$ versus $q$ (in unit of $m_{\pi}$ ) at density $\rho_{0}$ and $g^{\prime}=0$ (dotted line), and $g^{\prime}=0.6$ (solid line).

Figure 4. Pion dispersion relation in nuclear matter in the case $\rho=\rho_{0}, g^{\prime}=0$. The axes are in unit of $m_{\pi}$. The hatched regions indicate the region where the imaginary part does not vanish: the lower part is for $p h$ excitation the upper part is for $\Delta h$ excitation.

Figure 5. The same as Fig. 4 but $g^{\prime}=0.6$. The letters indicate the corresponding dotted lines.

Figure 6. Pion dispersion relation by eq. $(20,21), g^{\prime}=0.6$. The dashed line is for free pion.

Figure 7. Pion spectral function $A_{\pi}(\omega, q)$ (in the unit of $m_{\pi}^{2}$ ) versus $\omega$ (in unit of $m_{\pi}$ ), for $q=2.5 k_{F}, \rho=\rho_{0}$. Dotted line $g^{\prime}=0$; solid line $g^{\prime}=0.6$, the polarization by eq. (19); dashed line $g^{\prime}=0.6$ and the polarization by eq. (21). 